\title{
¿Qué podemos aprender de los estudios de caso?
}

Que peut-on apprendre des études de cas?

What can we learn from the case studies?

Robert D'Ercole, Sébastien Hardy et Jérémy Robert

\section{OpenEdition \\ Journals}

Édition électronique

URL : http://journals.openedition.org/bifea/2344

DOI : $10.4000 /$ bifea.2344

ISSN : 2076-5827

Éditeur

Institut Français d'Études Andines

Édition imprimée

Date de publication : 1 décembre 2009

Pagination : $573-591$

ISSN : 0303-7495

Référence électronique

Robert D’Ercole, Sébastien Hardy et Jérémy Robert, «¿Qué podemos aprender de los estudios de caso? », Bulletin de l'Institut français d'études andines [En ligne], 38 (3) | 2009, mis en ligne le 29

novembre 2013, consulté le 18 novembre 2020. URL : http://journals.openedition.org/bifea/2344 ; DOI : https://doi.org/10.4000/bifea.2344

\section{(c) $(1) \odot$}

Les contenus du Bulletin de l'Institut français d'études andines sont mis à disposition selon les termes de la licence Creative Commons Attribution - Pas d'Utilisation Commerciale - Pas de Modification 4.0 International. 


\title{
¿Qué podemos aprender de los estudios de caso?
}

\author{
Robert D'Ercole* \\ Sébastien Hardy** \\ Jérémy Robert***
}

\section{Resumen}

Esta síntesis propone una lectura global y comparada de los siete eventos presentados en este número temático. Esta se basa sucesivamente en un análisis de las consecuencias de estos eventos, en el descubrimiento de las vulnerabilidades estructurales o coyunturales que los originaron, y en las incidencias, a más o menos largo plazo, en materia de prevención de riesgos, de las crisis que estos eventos han provocado. Varias enseñanzas surgen del análisis y de la comparación de las tres aglomeraciones urbanas. La intensidad y la duración de las crisis no están relacionados con la magnitud del fenómeno físico; estas dependen sobre todo de la manera con la cual han sido afectados los elementos esenciales del funcionamiento del territorio. Además, descifrar la multiplicidad y las articulaciones de las vulnerabilidades para comprender las razones de la ocurrencia de un accidente o de un desastre y las modalidades de su gestión, refuerza el interés por un enfoque territorial y sistémico de los riesgos y de las crisis.

Palabras clave: estudios de caso, accidentes y desastres, consecuencias, crisis, vulnerabilidades estructurales, vulnerabilidades coyunturales, reducción de las vulnerabilidades

* Institut de Recherche pour le Développement (IRD), UR 029, programa Pacivur, Calle Teruel 357, Miraflores, Casilla 18-1209, Lima 18,Perú. E-mail: robert.dercole@ird.fr

** Institut de Recherche pour le Développement (IRD), UR 029, programa Pacivur, Av. Hernando Siles 5290 esq. calle 7, CP 9214 Obrajes, La Paz, Bolivia. E-mail: sebastien.hardy@ird.fr

*** Institut Français d'Études Andines (IFEA, UMIFRE 17, CNRS-MAEE)-Université de Savoie, CISM, Laboratorio EDYTEM (CNRS). E-mail: Jeremy.Robert@univ-savoie.fr 


\title{
Que peut-on apprendre des études de cas?
}

\section{Résumé}

Cette synthèse propose une lecture globale et comparée des sept évènements présentés dans ce numéro thématique. Elle s'appuie successivement sur une analyse des conséquences de ces évènements, sur la mise à jour des vulnérabilités structurelles ou conjoncturelles qui en sont à l'origine, et sur les incidences, à plus ou moins long terme, en matière de prévention des risques, des crises que ces évènements ont provoquées. Plusieurs enseignements sont tirés de l'analyse et de la comparaison des trois agglomérations urbaines. L'intensité et la durée des crises ne sont pas fonction de l'ampleur du phénomène physique en jeu ; elles dépendent surtout de la manière dont les éléments essentiels du fonctionnement du territoire ont été affectés. Par ailleurs, décrypter la multiplicité et les articulations des vulnérabilités en action pour comprendre les raisons de la survenue d'un accident ou d'une catastrophe et les modalités de leur gestion, souligne l'intérêt d'une approche territoriale et systémique des risques et des crises.

Mots clés : études de cas, accidents et catastrophes, conséquences, crises, vulnérabilités structurelles, vulnérabilités conjoncturelles, réduction des vulnérabilités

\section{What can we learn from the case studies?}

\begin{abstract}
This summary presents a global and comparative analysis of the seven events presented in this thematic issue. It is based on an analysis of the consequences of these events; the updating of structural or conjunctural vulnerabilities causing such events; and the impacts, on the medium and long run, in terms of risk prevention of the crises caused by these events. Several lessons have been learned from the analysis and comparison of the three urban agglomerations in Quito, La Paz and Lima. The intensity and duration of the crises are not a function of the degree of the physical phenomenon; they specifically depend on how the essential elements of the territory's operation have been affected. Moreover, decrypting the multiplicity and articulations of the vulnerabilities in order to understand why an accident or disaster occurs, and how they are managed, underlines the importance of a territorial and systemic approach to the risks and crises.
\end{abstract}

Key Words: case studies, accidents and disasters, consequences, crises, structural vulnerabilities, conjunctural vulnerabilities, vulnerability reduction

\section{INTRODUCCIÓN}

Los eventos analizados para dar cuenta de situaciones críticas vividas en La Paz, Lima y Quito son muy variados: son sucesos más o menos antiguos, de origen natural o antrópico, de diferente magnitud, que han afectado directa o indirectamente espacios de diferentes tamaños. Son ejemplos representativos de los accidentes y desastres ocurridos en las tres capitales durante los últimos 20 años. Son relativamente moderados en términos de pérdidas humanas, a pesar de su carácter a veces espectacular y de la extensión de su impacto territorial. 
Durante estas últimas décadas, las tres ciudades no han conocido eventos de gran magnitud que originasen un gran número de heridos o de muertos. Las condiciones del manejo de crisis no son pues las mismas que aquellas que movilizan medios colosales para llevar asistencia a numerosos heridos o para retirar rápidamente grandes volúmenes de escombros con la finalidad de encontrar sobrevivientes. Los eventos de Lurigancho-Chosica de 1987 (huaycos) y de La Paz en 2002 (inundaciones) se acercan a este tipo de situación, con un número de víctimas relativamente escaso. No es el caso de otros eventos cuyas características son diferentes. Sin embargo, algunos de estos eventos han provocado unas verdaderas crisis a escala de las aglomeraciones urbanas¹, revelando el carácter activo de la vulnerabilidad.

En este contexto, las lecciones que se extraen de los estudios de caso se articulan en torno a tres temas:

- En primer lugar, las consecuencias de los eventos: se trata de mostrar que estas deben ser abordadas siguiendo enfoques complementarios, lo que ayuda a comprender por qué algunos de estos eventos han podido desembocar en graves crisis mientras que otros han tenido un impacto más débil, a pesar de producir daños importantes.

- En un segundo momento, el análisis distingue las vulnerabilidades estructurales (o de fondo) de aquellas denominadas coyunturales, que remiten a circunstancias particulares. El análisis de las vulnerabilidades de fondo permite comprender las causas profundas del accidente o del desastre. Estas están ligadas a las opciones de desarrollo (Cartier et al., 2009) y dependen de dimensiones sociales, institucionales o técnicas. Dependen también de las condiciones de vulnerabilidad espacial y de la vulnerabilidad del sistema territorial. El análisis del manejo de crisis de los diferentes eventos permite registrar luego los problemas encontrados en el momento en que ocurrieron y descubrir las vulnerabilidades coyunturales más directamente ligadas al evento. Así, debemos considerar las causas inmediatas del desastre.

- Finalmente, el análisis de las incidencias de los eventos permite evaluar la capacidad de reacción y de adaptación de la sociedad urbana involucrada. Esta capacidad es reconocible mediante la implementación de dispositivos de reducción de las vulnerabilidades después del evento. Así se pueden establecer grados de incidencia, basados en la calidad de estos dispositivos.

En cada una de estas tres dimensiones del análisis, el propósito es poner en evidencia los puntos comunes y, en la medida de lo posible, las especificidades de cada ciudad. La comparación, aspecto importante de este balance, es posible gracias a una metodología común utilizada para la lectura de cada evento.

1 Las crisis de las que hablamos están ligadas a eventos mayores causados por fenómenos destructores de origen natural o antrópico que afectan a las sociedades y a los territorios. Estas pueden ser definidas como rupturas o muy fuertes perturbaciones, en dimensiones materiales o logísticas, pero también económicas, sociales o sanitarias. Ver el artículo de R. D’Ercole y P. Metzger (pp. 893-915) en este volumen. 


\section{DE LAS CONSECUENCIAS DE LOS EVENTOS A LA CRISIS}

Para captar su alcance en términos de crisis, las consecuencias de los eventos ocurridos pueden ser abordadas de diferentes maneras (cuadro 1).

El enfoque clásico de las consecuencias establece el balance de las víctimas (muertos y damnificados de todo tipo, desde heridos hasta personas que sufren indirectamente algunas perturbaciones), de los daños materiales y de los efectos sobre las actividades y servicios. Este es el tipo de información que se encuentra habitualmente en las bases de datos sobre las consecuencias de desastres².

En los ejemplos analizados, el número total de muertos se eleva a 200 aproximadamente, casi todos registrados en Lurigancho-Chosica (1987) y La Paz (2002). En conjunto, el número de personas damnificadas directa o indirectamente es elevado y, exceptuando los casos de Chillogallo (2003) y de la MIRR3 (2007), los servicios y actividades fueron muy afectados y a veces interrumpidos de manera prolongada. Los daños materiales son considerables en 4 casos, moderados en los otros. Por lo tanto, el enfoque clásico de las consecuencias resalta la importancia de tres eventos: La Paz (2002), Lurigancho-Chosica (1987) y Pichincha (19981999), aun cuando en este último caso el número de fallecidos es muy escaso 4 . Los eventos de Pampahasi (2008), del Trébol (2008) y de la MIRR (2007) se caracterizan por la importancia marcada de algunos tipos de consecuencias solamente. El incendio de Chillogallo (2003), a pesar de su carácter espectacular, presenta consecuencias moderadas en todos los campos.

El segundo enfoque de las consecuencias se refiere a los aspectos espaciotemporales de los eventos. Se puede considerar que mientras más dura el tiempo de resolución de los principales problemas (asistencia a las víctimas, restablecimiento de las vías de comunicación y del abastecimiento de agua potable, etc.), más intensa resulta la crisis. De igual manera, mientras más vasto es el territorio afectado por el evento, más compleja y difícil de manejar se vuelve la crisis. En tres de los casos analizados, el tiempo de resolución de los problemas fue largo (varios meses) y los espacios afectados fueron particularmente extensos, involucrando al conjunto de la aglomeración, en el caso del Pichincha (19981999), o a gran parte del territorio en los casos de La Paz (2002) y de Pampahasi (2008). Los demás eventos presentan efectos espaciales a veces extensos, pero tiempos más cortos de resolución de los problemas; esto se nota particularmente con los casos de Chillogallo (2003) y de la MIRR (2007).

La lectura del enfoque espacial de las consecuencias (cuadro 1), muestra un desfase notable entre la extensión geográfica de las zonas afectadas por la crisis y

2 En realidad, estas bases de datos ponen énfasis en el número de muertos, heridos, damnificados y en los daños materiales. Muy rara vez presentan una evaluación del costo de los daños. Generalmente brindan poca información sobre la manera en que fueron afectados las actividades y los servicios.

3 Margen Izquierda del Río Rímac (Cercado de Lima, Lima).

4 Además, es imputable a accidentes indirectamente ligados a la ceniza volcánica (caídas de personas desde los techos donde intentaban retirar la ceniza). 
aquellas que, al inicio, fueron afectadas por el (los) fenómenos(s) que originaron la crisis. Mientras que el espacio afectado fue importante desde el inicio en La Paz (2002) y en Quito (1998-1999), fue más bien de tipo puntual en los casos de Pampahasi (una canalización de transporte de agua), el Trébol (un intercambiador vial) y Chillogallo (una tubería de transporte de gasolina). Pero en resumidas cuentas, ello no impidió que los efectos se hicieran sentir sobre vastos espacios.

El enfoque de las consecuencias abordado sobre un plano sistémico permite comprender esta situación y el encadenamiento de fenómenos que han podido producirse a partir del momento en que al menos un elemento esencial del funcionamiento del territorio ha sido afectado. Exceptuando la MIRR, en todos los casos han habido elementos esenciales que fueron perturbados, dañados o destruidos. Por ejemplo vías de comunicación de primera importancia (LuriganchoChosica, 1987; La Paz, 2002) o elementos fundamentales de la red vial (El Trébol, 2008). En Quito se trata del aeropuerto. Sucedió lo mismo con aspectos esenciales del abastecimiento de agua (Pampahasi, 2008) o de energía (Lurigancho-Chosica, 1987; Chillogallo, 2003). A través de estos ejemplos se observará la importancia de las redes, aunque no todos los elementos esenciales del territorio afectado pertenezcan a ellas (por ejemplo los establecimientos de salud, víctimas de la penuria de agua en el caso de Pampahasi, 2008). Como lo señala el cuadro 1, si los problemas que confrontan los elementos esenciales no se resuelven a tiempo, se propagan al conjunto del sistema en el cual se sitúan (caso de gran parte del sistema de distribución de agua potable afectado en La Paz —Pampahasi, 2008o de la red vial en Quito, en 2008). Otros sistemas también pueden ser afectados, prolongando así la cadena de fallas, perturbaciones y destrucciones. Por ejemplo la caída de ceniza sobre Quito afectó — directa o indirectamente- a numerosas empresas económicas de la ciudad. El accidente del Trébol provocó la parálisis de las vías de comunicación que dependían del intercambiador, y a eso se debió el cierre de los establecimientos escolares, el mismo que, a su vez, originó problemas en la economía de la capital ecuatoriana (por ejemplo en razón del ausentismo en las empresas de muchos padres de familia). Los encadenamientos de dificultades ligadas a la multiplicación de subsistemas territoriales damnificados han sido particularmente numerosos en La Paz en 2002. Como una cosa lleva a la otra, se ven afectados importantes sectores de la actividad económica y social de las aglomeraciones, en espacios cada vez más vastos. Todo ello desemboca en un estado de crisis cada vez más intensa.

Se puede considerar que, en 4 casos al menos, el sistema territorial fue afectado por este proceso de encadenamiento de fenómenos. En los demás casos, el proceso pudo ser rápidamente contenido, como en Chillogallo donde el daño del poliducto no tuvo sino un efecto limitado sobre la distribución de gasolina a escala de la aglomeración 5 .

5 Una perturbación que duró 5 horas, según J. Estacio. 
Robert D’Ercole, Sébastien Hardy, Jérémy Robert
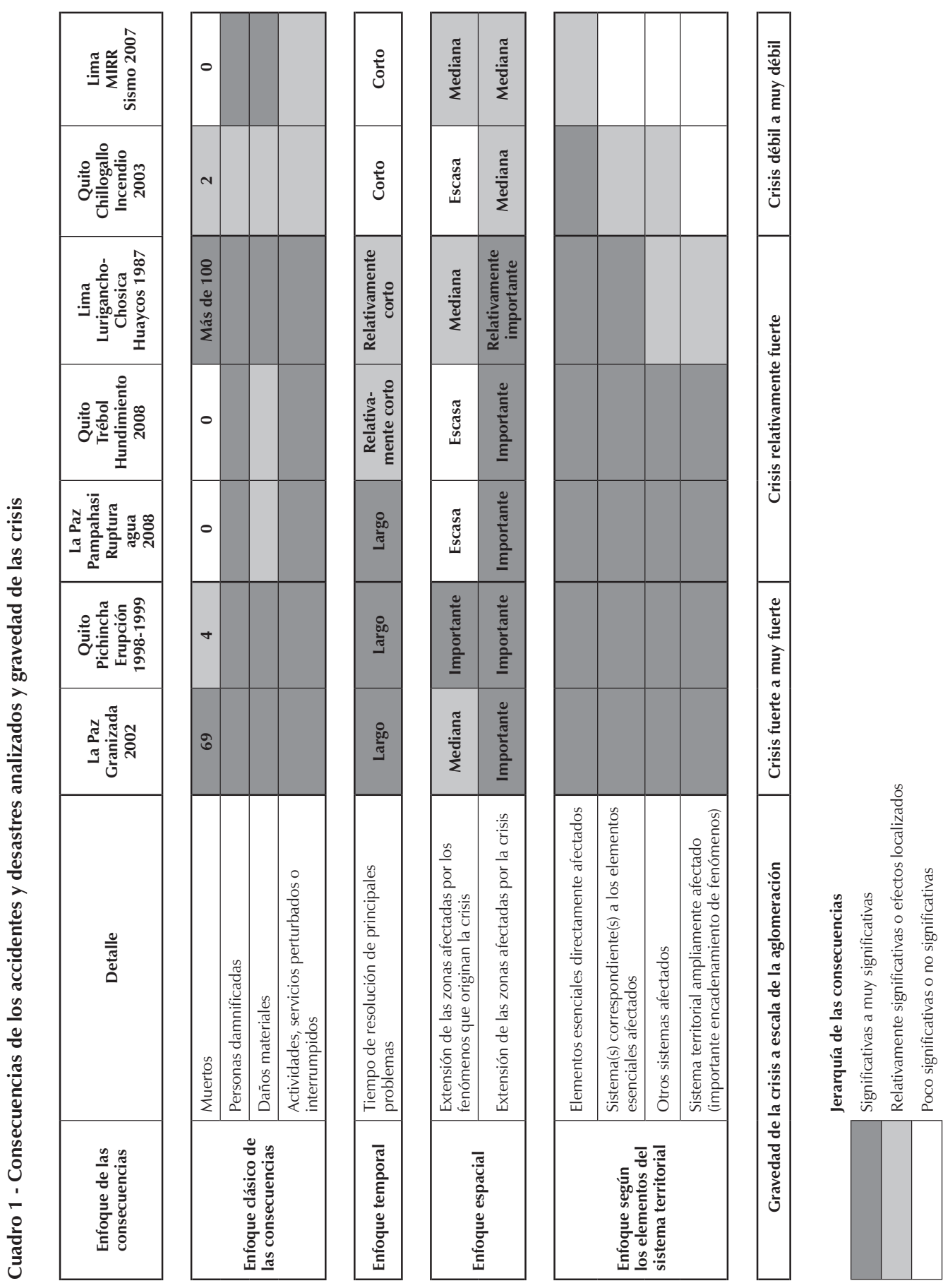
El conjunto de los enfoques de consecuencias permite clasificar los eventos según la gravedad de la crisis. Evidentemente, los eventos de La Paz (2002) y de Quito (Pichincha, 1998-1999), han originado las crisis más graves, tanto por la importancia de los daños como por la duración de las perturbaciones, la extensión de los sectores afectados y el encadenamiento (o efecto dominó) de fenómenos dañinos. En Pampahasi (2008), El Trébol (2008) y Lurigancho-Chosica (1987) las crisis fueron fuertes, pero de menor grado que en los casos precedentes, en razón de daños más moderados (Pampahasi, El Trébol) o de un tiempo de resolución más corto de los problemas (El Trébol, Lurigancho-Chosica). Cabe señalar que el evento de Lurigancho-Chosica fue un verdadero desastre local, pero su impacto sobre la aglomeración fue menos intenso. Por último, en Chillogallo y en la MIRR, las crisis pueden ser consideradas débiles a escala de la aglomeración, ya que el evento permaneció limitado a los espacios inicialmente afectados, con una explicación que difiere según los casos: en el primero, una intervención relativamente rápida y eficaz de los socorros, seguida del arreglo del poliducto; en el segundo, prácticamente ninguna intervención exterior a la zona afectada, y la crisis fue resuelta con los medios locales.

\section{VULNERABILIDADES ESTRUCTURALES (O VULNERABILIDADES DE FONDO)}

Las vulnerabilidades estructurales (o de fondo) ligadas a las bases de la sociedad y a los modos de desarrollo han sido enfocadas de 5 maneras complementarias: vulnerabilidad espacial, societal, institucional, técnica y propia del territorio considerado como sistema (cuadro 2). Cada forma engloba varios factores de vulnerabilidad y el balance de los siete eventos traduce la multiplicidad de las vulnerabilidades que originan fenómenos perturbadores o destructores y sus consecuencias. Varios de estos factores interactúan (o actúan al mismo tiempo) produciendo, según las combinaciones, situaciones inéditas, a veces muy complejas. Cada evento posee así su propia gama de vulnerabilidades, aunque son observables algunos puntos comunes.

Por lo tanto, si la exposición a las amenazas (el incendio, la inundación, la erupción volcánica, etc.) constituye el factor de vulnerabilidad común, el que ha desencadenado la crisis no es forzosamente el primer responsable de ella. Las dificultades de movilidad y de accesibilidad son otros factores de vulnerabilidad espacial6 que plantean a veces problemas estructurales, en particular en La Paz debido a su topografía y su contexto geomorfológico.

Los factores societales cubren aspectos socioeconómicos, sociales, culturales, así como la percepción del riesgo. El componente socioeconómico resalta en la

6 La noción de vulnerabilidad espacial ha sido explicitada en D’Ercole y Metzger, 2004. Esta permite distinguir, dentro de un territorio, espacios más o menos frágiles según características espaciales, como la exposición a las amenazas y la accesibilidad de los espacios, características espaciales retomadas aquí. 
mayoría de los estudios de caso y constituye un problema de fondo común a las tres capitales. Por falta de políticas de vivienda adecuadas, la población más desfavorecida no tiene con frecuencia otra alternativa para albergarse sino ocupar los terrenos menos caros, pero al mismo tiempo menos aptos para la urbanización. En Lurigancho-Chosica, Chillogallo o en la MIRR, las poblaciones desfavorecidas son las que han sufrido por la necesidad de instalarse sobre terrenos que presentaban peligros. El aspecto social está especialmente marcado en dos casos: en La PazPampahasi (problemas planteados por la comunidad rural de Hampaturi por el acceso a las canalizaciones deterioradas) y en la MIRR (población históricamente marginalizada y en conflicto con las autoridades). En Quito, la práctica muy antigua del relleno de las quebradas, que sigue lógicas no solo técnicas (Sierra, 2000), puede ser considerada como un factor cultural de vulnerabilidad. En La Paz, S. Hardy subraya el rol del vestuario de las comerciantes callejeras indígenas en la mortalidad ligada a las inundaciones de 2002. La calidad de la percepción del riesgo también tiene un papel importante en las características, e incluso en la intensidad de la crisis. A veces esto se debe a la antigüedad de las experiencias anteriores o a su ausencia (Quito y el Pichincha, Lurigancho-Chosica, Chillogallo). En la MIRR, como concurren numerosos riesgos sociales (desempleo, problemas de salud infantil, delincuencia, etc.), los riesgos de origen natural pasan a un segundo plano.

A menudo los análisis han revelado una vulnerabilidad institucional que tiene que ver con las dificultades relacionales entre organismos públicos o entre estos últimos y la población, con problemas jurídicos y con el difícil control de la ocupación del suelo. Los problemas de fondo que existen entre la municipalidad de Quito y PETROECUADOR o entre la municipalidad de Lima y la población de la MIRR han tenido un impacto en las crisis vividas en Quito (Chillogallo, 2003) y en Lima (MIRR, 2007). En este último caso, la inadecuada gestión del riesgo se asocia a problemas jurídicos ligados a la ocupación del suelo. En La Paz (Pampahasi, 2008) también se destaca este aspecto. Aquí, la imprecisa situación legal de una empresa responsable por delegación de un servicio público (el agua potable) creó disfunciones continuas que, por acumulación, amplificaron enormemente la situación de crisis. El control de la ocupación del suelo en relación con la existencia de riesgos ha planteado problemas en todos los casos analizados. En La Paz, por ejemplo, el Gobierno Municipal emite ordenanzas para reglamentar el uso de la propiedad en las zonas expuestas a los riesgos. Sin embargo, basándose en la independencia de las jurisdicciones, la institución nacional encargada de otorgar títulos de propiedad (Derechos Reales), no integra las restricciones municipales en materia de exposición a los riesgos. La situación es idéntica en Lima. Por no intercambiar y construir puentes entre ellas, el funcionamiento de estas instituciones contribuye también a la vulnerabilidad de fondo de las capitales andinas.

Otras vulnerabilidades estructurales tienen que ver con factores técnicos, aun cuando en algunos casos es posible establecer vínculos entre estos últimos y las capacidades financieras locales. La falta de instrumentos de previsión o de alerta es uno de los factores técnicos identificados. No existía ningún medio de este 
tipo en Lurigancho-Chosica para evacuar a la población y reducir el número de víctimas ligadas a los huaycos. En La Paz, la dificultad de los científicos para prever las tempestades de granizo radicó en la obsolescencia de la red de observación. La ciudad de Quito, por su parte, no dispone de sistemas eficaces y rápidos para desviar la circulación en caso de obstáculo: un problema de control del tráfico que hizo más compleja la crisis ligada al Trébol. En el mismo orden de ideas, no existía ningún sistema de detección de fugas de combustibles en Chillogallo. Más a menudo, se han resaltado serias deficiencias técnicas: la mala calidad de la construcción en la MIRR, la antigüedad de las instalaciones en Chillogallo o la rápida saturación de los sistemas de evacuación de las aguas en La Paz o en Quito. Como telón de fondo, generalmente lo que hace falta son los conocimientos científicos o técnicos. En Quito, los conocimientos científicos sobre el comportamiento del volcán Pichincha eran manifiestamente insuficientes para manejar la crisis en buenas condiciones. También en Quito, los conocimientos ligados a un riesgo aún poco considerado, el tecnológico, son aún parciales y poco tomados en cuenta por las autoridades públicas. En La Paz, la ceguera de los gestores de la crisis de Pampahasi que consideraban el evento como un simple accidente de un elemento del sistema de producción de agua potable puede ser percibido como una falta de conocimiento, por no decir de sensatez. Este accidente no podía ser resuelto, ni anticipado, sino saliendo de la esfera técnica y reflexionando en términos de transmisión de vulnerabilidades de un sistema a otro7.

Los últimos factores de vulnerabilidad dependen del territorio considerado como sistema. Si se considera un sistema territorial como un conjunto de elementos en interacción, es importante identificar los principales y comprender cómo pueden ser capaces de perturbar el funcionamiento y desarrollo del conjunto. Ocurre cuando determinado elemento presenta debilidades intrínsecas o cuando está expuesto a una amenaza. Si es dañado o destruido, las consecuencias sobre el sistema urbano serán tanto más fuertes si su importancia para el territorio es grande, si el número de elementos que dependen de él es elevado y si las alternativas para remplazarlo son poco numerosas. Y ello puede ser previsto y estudiado ( $D^{\prime}$ Ercole \& Metzger, 2004). La situación puede ser todavía más penalizante para el sistema territorial cuando el espacio afectado por una amenaza concentra varios de estos elementos esenciales.

Durante las inundaciones de 2002, todos estos factores estuvieron presentes en La Paz. Varios elementos mayores del funcionamiento territorial, en su mayoría concentrados en el centro de la ciudad a lo largo de las principales vías de comunicación, fueron dañados, provocando una cadena de consecuencias por dependencias y ausencia de alternativas (en particular en el campo de la comunicación vial). La situación fue del mismo tipo en Lurigancho-Chosica (1987), donde una central eléctrica, una planta de tratamiento de agua y una carretera esencial para el abastecimiento alimentario de Lima estaban expuestas a los huaycos y, lógicamente, fueron afectadas por ellos. En los demás casos, al menos un 
Robert D'Ercole, Sébastien Hardy, Jérémy Robert

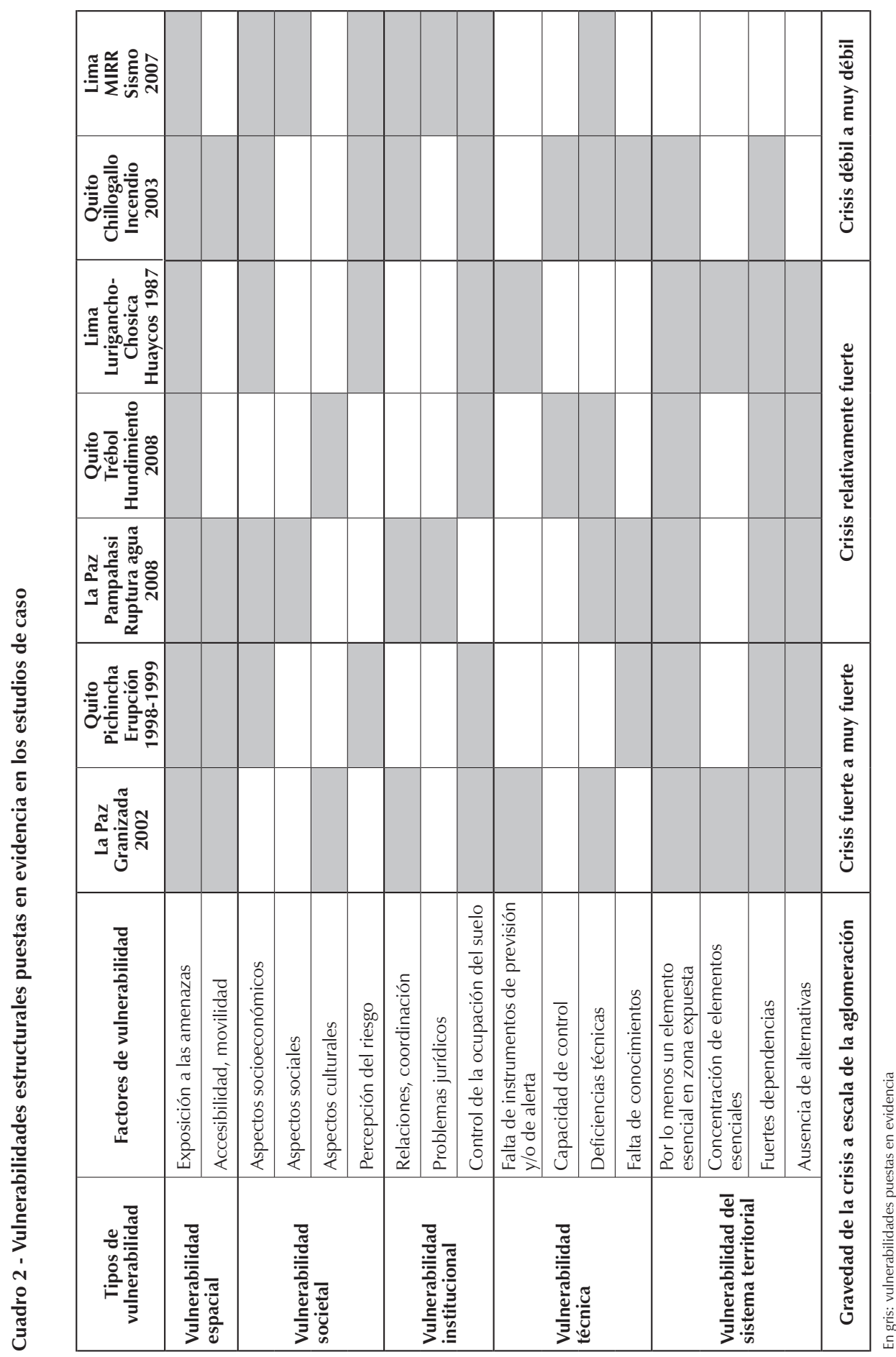


elemento esencial estuvo en situación de exposición y fue afectado (el aeropuerto, y el intercambiador vial del Trébol en Quito, la planta de tratamiento de agua de Pampahasi, etc.). Asociada a la ausencia de alternativas, la dependencia de los territorios a estos elementos siempre ha provocado numerosas consecuencias.

\section{VULNERABILIDADES COYUNTURALES}

A diferencia de las vulnerabilidades estructurales, las coyunturales están más directamente ligadas al evento en sí o a condiciones particulares del contexto en el cual este se produjo. Tres conjuntos de factores han sido considerados: contingentes, institucionales y ligados a la articulación entre espacios afectados y recursos (cuadro 3).

Los factores contingentes tienen que ver con todo aquello que es fortuito, ocasional, incierto. En otras palabras, atañen a fenómenos cuya ocurrencia o alcance no se controlan, pero que tampoco son del todo imprevisibles. Un evento clásico es el de las condiciones meteorológicas, generalmente presentadas en los informes como «excepcionales», que preceden a menudo a las inundaciones, los deslizamientos de tierra o los huaycos. Esto es lo que ocurrió en La Paz (2002), en Lurigancho-Chosica (1987) y en Quito (El Trébol, 2008). Las características físicas de los fenómenos pueden también ser factores contingentes que orientan de cierta manera las consecuencias, como el manejo de la crisis. La manera con que el incendio se propagó en Chillogallo entra en esta lógica. De haberse perforado el poliducto un poco más aguas arriba o abajo y/o bajo otras condiciones anemométricas, la situación hubiese sido diferente. En el caso del Pichincha, los productos volcánicos que podían amenazar directamente a la población, y sobre todo su volumen y extensión, causaron gran incertidumbre durante varios meses, a lo largo de los cuales se contemplaron varios escenarios. Las mismas lluvias de ceniza sobre Quito estaban ligadas a circunstancias meteorológicas particulares relacionadas con la orientación del viento.

El momento preciso en el que ocurre el evento es generalmente mencionado para explicar la naturaleza y la importancia de un accidente o de un desastre, en un sentido positivo o negativo. Por ejemplo, aun cuando el número de destrucciones totales de viviendas fue escaso en la MIRR, es posible considerar que el número de víctimas hubiese sido mayor si el terremoto había sorprendido a los habitantes de noche, en pleno sueño y no a las $18 \mathrm{~h} 40$. Pero esto también puede funcionar en un sentido negativo. J. Estacio destaca la ausencia de reactividad de la población afectada por el incendio de Chillogallo ocurrido a las 3 de la madrugada. Sin embargo, las condiciones de movilidad para enviar a los auxilios fueron buenas, lo que no hubiera sido el caso en pleno día. Por su lado, S. Hardy muestra que la hora de la inundación de 2002 (entre 14 h20 y 15h45) es uno de los elementos que indujo los mayores daños y dificultades para el manejo de crisis (importancia del tráfico, familias separadas, etc.).

Entre los demás factores contingentes figura el contexto político y social de ocurrencia del evento. En Quito, la declaración de la alerta amarilla el $1^{\circ}$ de octubre 
de 1998 coincidió con la presentación de medidas económicas impopulares por parte del presidente ecuatoriano. La población asoció los dos eventos, estimando que la alerta volcánica solo constituía una maniobra de diversión. En otros casos es un movimiento social. Así, en La Paz (Pampahasi) las comunidades rurales bloquearon el paso de acceso a las canalizaciones dañadas porque sus tierras de cultivo se habían inundado. En Lurigancho-Chosica, C. Abad enfatiza el papel de los robos y saqueos que ocurrieron durante el desastre, afectaron a la población damnificada y perturbaron el manejo de la crisis.

Los factores institucionales de vulnerabilidad han sido considerados ya como factores estructurales, pero pasan a ser coyunturales cuando toman forma en el momento de la crisis aun cuando las dificultades institucionales de fondo los hacían previsibles. Es así como las debilidades de las relaciones institucionales en tiempo normal se ponen de manifiesto durante la crisis a través de numerosas dificultades de coordinación entre instituciones y de relaciones entre las instituciones y la población. En varios casos, como en los eventos analizados en La Paz y en Lima, estas dificultades se tradujeron sobre todo por una ausencia de coordinación general de los actores del manejo de crisis y por una falta de estrategia operacional de intervención. Bajo este aspecto, Quito parece mejor armado en la medida en que estos temas no han constituido mayor problema en el manejo de las tres crisis ocurridas en esta ciudad. En cambio, la gestión de las alertas ha sido uno de los principales puntos débiles de la crisis provocada por la erupción del Pichincha. Durante numerosos meses, los científicos y las autoridades fueron incapaces de controlar esta herramienta, pues las alertas estuvieron desfasadas en relación con la actividad del volcán. La dificultad para juzgar la evolución o el fin de la crisis también ha sido retenida como factor coyuntural de vulnerabilidad institucional. En Quito esto está ligado a la duración de la actividad volcánica y a la dificultad de mantener en alerta a una población que se fue acostumbrando a vivir con un volcán en actividad, pero en apariencias poco peligroso. También en Quito, el reinicio de las clases fue decidido demasiado rápido, cuando los principales problemas de movilidad ligados al hundimiento del Trébol no habían sido resueltos todavía. En La Paz (Pampahasi, 2008), la complejidad de la crisis desconcertó a sus gestores, quienes pensaron poder resolverla mediante soluciones técnicas (también complejas en sí), subestimando, por lo menos en un primer momento, los problemas sociales y jurídicos planteados por la situación.

Para evaluar las vulnerabilidades de manejo de crisis, también se ha reflexionado sobre la articulación entre espacios afectados y espacios recursos del manejo de crisis, partiendo de la hipótesis según la cual un buen manejo de crisis está ligado a la calidad de este vínculo8. Así los problemas de movilidad y de accesibilidad fueron frecuentes, intensificaron las crisis e hicieron más complejo su manejo (por ejemplo, vías importantes interrumpidas en La Paz o en Lurigancho-Chosica; un puente destruido en Chillogallo, el aeropuerto de Quito cerrado, etc.). El acceso a recursos esenciales como los servicios de salud o el agua potable fue problemático

8 Para mayor información sobre este tema, ver R. D’Ercole y P. Metzger en este volumen, pp. 893915. 
¿Qué podemos aprender de los estudios de caso?

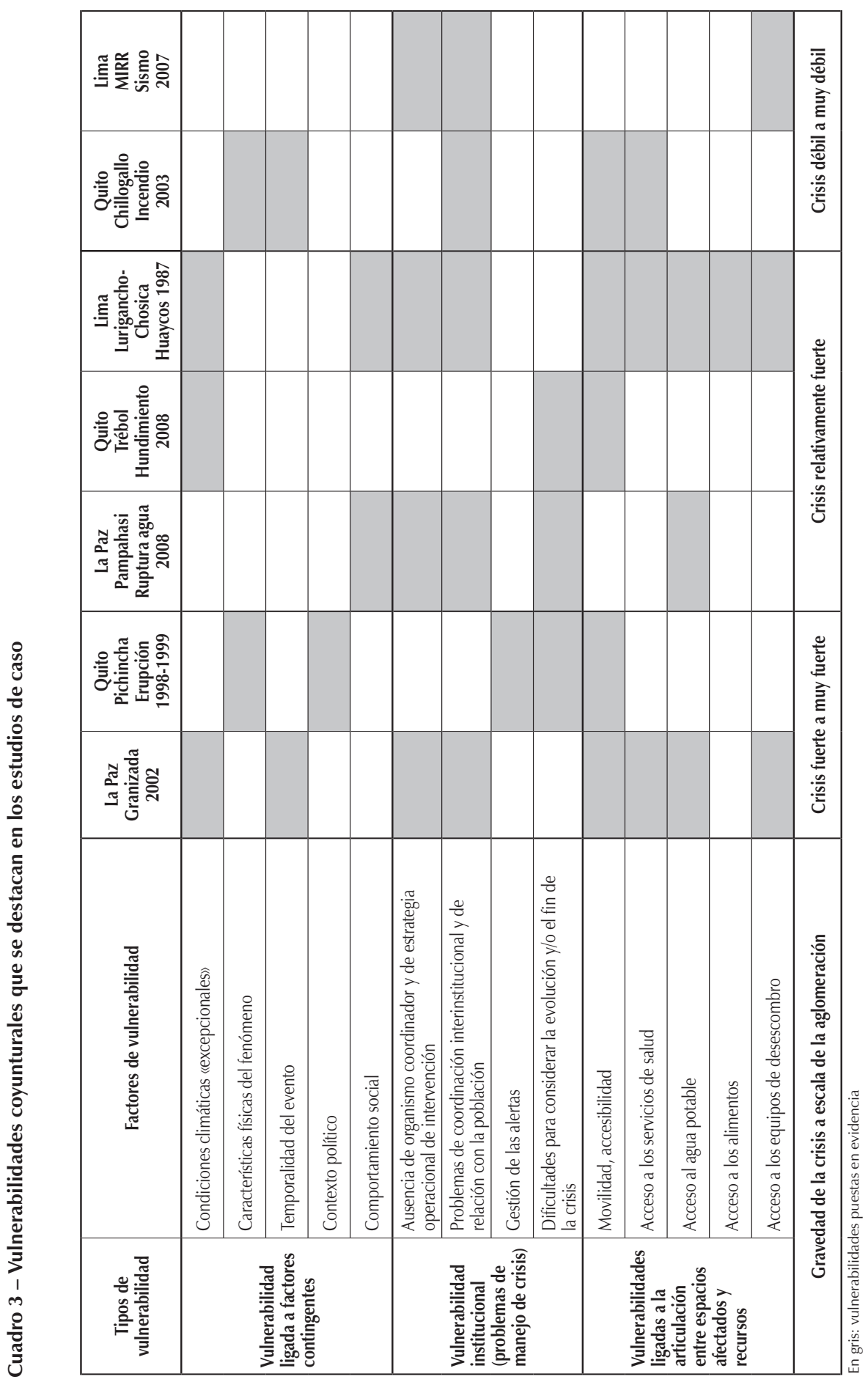


en varios casos, particularmente en La Paz (2002) o en Lurigancho-Chosica donde se observaron dificultades en ambos campos. En 2008, la población, los establecimientos de salud y las empresas sufrieron racionamientos de agua durante la crisis de Pampahasi (2008), mientras que en Chillogallo parece haber planteado problemas el recurso referido a la atención médica. El evento hizo tomar conciencia que la ciudad de Quito no disponía de servicios hospitalarios suficientes para personas quemadas en alto grado. Generalmente el acceso a los alimentos no generó mayores problemas, salvo en Lurigancho-Chosica donde fueron señaladas dificultades tanto localmente (desorganización en la distribución de alimentos) como a escala metropolitana (interrupción de una vía de comunicación mayor que permitía el abastecimiento en alimentos de la capital).

\section{INCIDENCIAS DE LOS EVENTOS}

Las incidencias de los desastres no son siempre negativas y pueden constituir oportunidades de modificación, a veces radical, de las situaciones que les dan origen, a fin de reducir las vulnerabilidades y mejorar las condiciones del desarrollo (Lavell, 2000). Eso fue lo que ocurrió tras la erupción del Nevado del Ruiz en Colombia, en 1985, y la destrucción muy mediatizada de la ciudad de Armero. El evento dio origen a una verdadera toma de conciencia de la necesidad de reducir los riesgos y sus efectos sobre el funcionamiento y el desarrollo de las sociedades. Esto se concretizó en particular a través de una mejora sustancial del sistema de defensa civil colombiano y de una gestión preventiva de los territorios en algunas ciudades, sobre todo en Bogotá. En los estudios de caso presentados, las crisis no alcanzaron la magnitud de la que Colombia conoció en 1985. Sin embargo, es posible, a pesar del carácter reciente de algunas, identificar incidencias positivas y negativas. De esta manera, los eventos han sido clasificados según la importancia de las incidencias en términos de cambios positivos, estableciendo al mismo tiempo una relación entre el grado de incidencia y la gravedad de la crisis (cuadro 4).

El grado de incidencia más escaso concierne a la MIRR, afectada por el sismo de 2007. Las mejoras han sido mínimas (capacitación de brigadistas), mientras que las vulnerabilidades anteriores al sismo siguen estando presentes e incluso se han acentuado. Las reparaciones han sido insuficientes y numerosas viviendas están fragilizadas y, por ende, más vulnerables que antes frente a un próximo terremoto. De manera general, el sismo no ha servido para mejorar la situación de los barrios de la MIRR. No se han adoptado medidas preventivas y el statu quo persiste asentando la vulnerabilidad de una población marginalizada de larga data y en conflicto latente con la municipalidad por la ocupación del suelo.

La incidencia de dos eventos (Chillogallo, 2003 y Pampahasi, 2008) puede ser considerada como escasa, aun cuando la gravedad de la crisis no es la misma en los dos casos.

En términos de incidencias, entre los activos de las crisis de Chillogallo están las respuestas de tipo técnico. La vigilancia de los poliductos ha sido mejorada y la instalación de sistemas de detección de fuga de combustible está en curso, 
así como el tratamiento de vulnerabilidades (en particular antigüedad de algunos elementos de la red). Se trata sobre todo de acciones efectuadas por la empresa PETROECUADOR. Por lo contrario, no se ha observado una evolución en el plano de la vulnerabilidad de la población, tanto de información preventiva como de una gestión de la ocupación del suelo que tomara en cuenta las amenazas. Como en el caso de la MIRR, pero en menor grado, la población de Chillogallo sigue estando marginalizada y vulnerable. El incendio de 2003, a pesar de haber sido uno de los más graves ocurridos en la capital ecuatoriana, no ha sido una oportunidad de cambio, como los habitantes de Chillogallo pudiesen haberlo esperado al día siguiente de su ocurrencia, con la visita del alcalde de Quito, del ministro de Energía y Minas y del presidente de PETROECUADOR.

La crisis de Chillogallo fue más bien débil a escala metropolitana porque estuvo a la vez localizada y tratada con cierta velocidad. Se puede entonces comprender la escasa incidencia de esta crisis, sobre todo en un contexto de marginalización de la población involucrada.

En cambio la crisis de Pampahasi, que perturbó a más de un tercio de la aglomeración paceña, afectando en particular a pobladores de nivel socioeconómico relativamente alto, no desembocó en incidencias significativas. Aquí también se dieron esencialmente respuestas técnicas (consolidación de los terrenos que soportaban canalizaciones, búsqueda de alternativas técnicas para la alimentación en agua). Sin embargo, los problemas de fondo que remitían a lo político-social (comunidades indígenas) y lo jurídico (situación de EPSAS) no han sido resueltos, lo que denota la dificultad de las autoridades de La Paz para afrontar temas en los que numerosos intereses están en juego.

Las incidencias son un poco más significativas en las crisis de Lurigancho-Chosica (1987) y de La Paz (2002). Por un lado, a semejanza de los casos precedentes, se ha hecho un esfuerzo sobre todo en los aspectos técnicos, con la construcción de numerosas obras de protección. Por otro lado, en ambos casos se han realizado estudios que desembocaron en mapas de amenazas y de riesgos9. Sin embargo, en Lurigancho-Chosica, la cartografía y las recomendaciones emitidas en términos de ocupación del suelo no han sido útiles en la medida en que las zonas afectadas por los huaycos de 1987 se ven nuevamente habitadas y que el mantenimiento de las obras de protección no está garantizado. En La Paz, por cierto, las acciones emprendidas son muy visibles y denotan cierta reactividad por parte de la municipalidad, pero no aportan verdadera solución a las vulnerabilidades de fondo: concentración de elementos esenciales del territorio en zona expuesta, peso de las dependencias, alternativas de funcionamiento insuficientes o inexistentes, dificultades en materia de accesibilidad, modalidades problemáticas de ocupación del suelo, exigencias socioeconómicas y culturales, etc. Sin embargo, cabe poner en exégesis un punto positivo, referido a la creación de un COE (Comité Operativo de Emergencias) municipal, que permite vislumbrar

9 Tanto en Lurigancho-Chosica como en La Paz, estos estudios han sido financiados por el PNUD (Programa de las Naciones Unidas para el Desarrollo). 


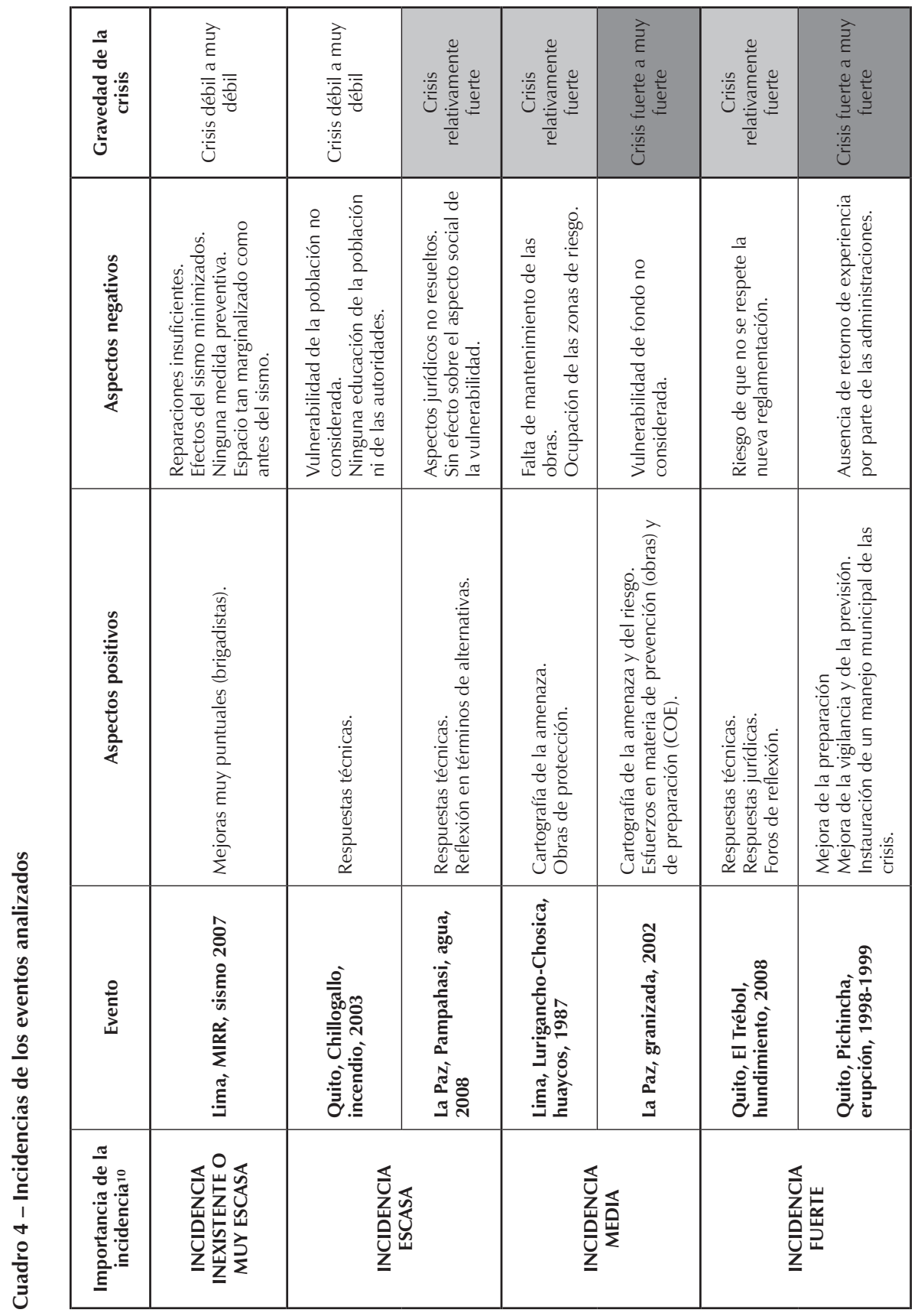

年 
un manejo más eficaz de las futuras crisis, siempre y cuando disponga de medios humanos, técnicos y financieros suficientes y adecuados.

Las incidencias más fuertes atañen a dos eventos ocurridos en Quito (El Trébol, 2008 y Pichincha 1998-1999) y, a pesar de la escasa incidencia de la crisis de Chillogallo, denotan una mejor capacidad de esta ciudad para sacar partido de eventos que han provocado daños. Ambos eventos son muy diferentes, por la extensión de los fenómenos al inicio y por la duración de la crisis, pero tanto uno como otro desembocaron en mejoras sensibles en términos de reducción de las vulnerabilidades. Por un lado, el evento de El Trébol produjo respuestas técnicas referentes al mejoramiento de las infraestructuras hidráulicas, pero también respuestas jurídicas a propósito del uso de terrenos edificados sobre rellenos de quebradas. Además, debates públicos, como los que organizó el Colegio de Arquitectos del Ecuador, permitieron adelantar soluciones para mejorar las condiciones de movilidad en la capital. Por su parte, la crisis ligada al reinicio de la actividad y la erupción del volcán Pichincha trajo consigo mejoras muy sensibles en términos de preparación de la población y de las administraciones, de coordinación entre instituciones, de vigilancia y de previsión volcánica y también de comportamiento de los medios de comunicación frente a este tipo de situaciones. Por otro lado, el evento dio origen a una verdadera institucionalización de la gestión de riesgos y del manejo de crisis en Quito. Como el liderazgo de esta gestión ha pasado de Defensa Civil al alcalde de Quito, este y sus sucesores, han hecho del tema de los riesgos una prioridad. La implementación de este dispositivo ha contribuido a un mejor manejo de las crisis posteriores, como aquel ligado a la erupción del Reventador en 2002 o aquel, desarrollado aquí, que concierne al Trébol (2008).

\section{CONCLUSIONES}

Esta síntesis de los siete estudios de caso permite extraer numerosas enseñanzas en materia de consecuencias de desastres, de vulnerabilidades y de incidencias, teniendo como telón de fondo la puesta en evidencia de puntos comunes entre eventos y entre ciudades. Algunas de estas enseñanzas, quizás las más significativas, son retomadas en esta conclusión.

La magnitud de las perturbaciones (o de las crisis) que una ciudad puede sufrir tras accidentes y desastres no está directamente ligada al origen natural o antrópico de estos eventos ni a su extensión espacial inicial. A escala metropolitana, eventos puntuales, como el que provocó el corte del abastecimiento de agua potable en La Paz en 2008 o el que afectó la movilidad de Quito ese mismo año (hundimiento del Trébol), originaron una crisis por lo menos tan fuerte como la que sacudió Lima con el desencadenamiento de huaycos en Lurigancho-Chosica en 1987. El análisis de las vulnerabilidades de fondo permite comprender esta situación. El punto común del conjunto de las crisis es la exposición a una amenaza de origen natural o generada por el hombre. La convergencia de varios factores de vulnerabilidad, cuya combinación difiere según el caso, marca así la naturaleza y la intensidad de 
la crisis, porque esta última, por definición, se alimenta de todas las debilidades existentes. Sin embargo, la crisis es siempre intensa cuando atañe a elementos esenciales del territorio y cuando estos últimos originan efectos en cadena ligados a las dependencias y a las ausencias de alternativas. Esta situación es característica de los medios urbanos, en razón de su complejidad y de la interconexión de numerosas redes11. La crisis permite también entender la necesaria distinción entre el mapa de la zona de impacto inicial (correspondiente al mapa de amenazas) y el mapa de las consecuencias potenciales (correspondiente al mapa de riesgo), ya que este último cubre un espacio generalmente mucho más extenso. La confusión existente entre estos dos tipos de mapas explica la dificultad de manejo de los riesgos y de las crisis de manera global y eficaz.

Otra enseñanza de los estudios tiene que ver con la dimensión temporal de las incidencias. A veces, las incidencias positivas de un evento grave se sienten bastante tiempo después del evento; tal es el caso de Lurigancho-Chosica, donde se han construido obras de protección (diques, presas filtrantes, etc.) tan solo 13 años después del desastre. Sin embargo, en el conjunto, las incidencias más sobresalientes aparecen muy rápidamente después del evento; por ejemplo tras las inundaciones de La Paz en 2002 o en Quito (crisis del Trébol y del Pichincha). Ello subraya la idea según la cual las oportunidades de cambio se deben asir rápidamente tras un desastre, pues de lo contrario la experiencia corre el riesgo de no ser aprovechada con miras a reducir las vulnerabilidades.

Por último en todas las crisis analizadas, existen incidencias positivas, aunque sean mínimas, de un evento dañino. Al mismo tiempo, las incidencias dependen a menudo de operaciones técnicas, resultantes de una tradición que privilegia la amenaza y, por lo tanto, las soluciones de ingeniería civil que buscan contenerla. Esta concepción técnica de las medidas de prevención está fundamentalmente ligada a un problema conceptual, en la medida en que el riesgo es generalmente asimilado a la amenaza.

En cambio, las incidencias más fuertes son aquellas que salen del marco estrecho de las soluciones técnicas para cubrir otros campos de la reducción de la vulnerabilidad: social, socioeconómico, institucional, jurídico, etc. Quito parece ser la ciudad más avanzada en este campo, reforzada por una municipalidad preocupada por la gestión de riesgos, a diferencia de Lima, por ejemplo. De manera general, la capital ecuatoriana goza de mayor experiencia gracias a los numerosos eventos que han sido aprovechados en términos de aprendizajes. Al mismo tiempo, con la municipalización de la gestión de crisis en un territorio urbano controlado por una sola autoridad político-administrativa12, Quito se ha dotado de medios para coordinar el manejo de las crisis y desarrollar una estrategia de intervención única sobre la totalidad del territorio13.

11 Sobre este tópico, ver el artículo de P. Metzger y R. D’Ercole, pp. 917-936.

12 A diferencia de La Paz (dos administraciones principales: La Paz y El Alto) y sobre todo de la aglomeración de Lima/Callao (49 distritos urbanos, cada cual con su alcalde).

13 Salvo el pequeño Cantón Rumiñahui, cuya gestión depende de otra administración. Ver el artículo de J. Robert, R. D’Ercole, P. Pigeon y T. Serrano, pp. 709-733. 
Sin embargo, sea cual fuere la ciudad, una de las principales debilidades radica en no considerar el territorio en la gestión de riesgos y en el manejo de las crisis, y en la valoración excesiva de uno o algunos de los aspectos de la cuestión en menosprecio de sus articulaciones. El enfoque sectorial y analítico incrementa la gravedad de las crisis. Reduce las enseñanzas que se pueden extraer de estos eventos y limita su alcance en términos de política de prevención. Por consiguiente, hemos de hacer un esfuerzo en materia de capacitación de investigadores y de gestores territoriales para desarrollar una visión territorial y sistémica de los riesgos y de las crisis.

\section{Referencias citadas}

CARTIER, S., VINET, F. \& GAILLARD, J.-C., 2009 - Maître du monde ou maître de soi ? In: Risques et environnement : recherches interdisciplinaires sur la vulnérabilité des sociétés (S. Becerra \& A. Peltier, eds.): 9-20; París: Éditions L’Harmattan.

D'ERCOLE, R. \& METZGER, P., 2004 - Vulnerabilidad del Distrito Metropolitano de Quito, 496 pp.; Quito, Ecuador: MDMQ, IRD. Colección Quito Metropolitano.

LAVELL, A., 2000 - Desastres y Desarrollo: Hacia un Entendimiento de las Formas de Construcción Social de un Desastre: El Caso del Huracán Mitch en Centroamérica, 25 pp.; Lima: La Red. 
